\title{
Pigment Epithelium-Derived Factor Regulates Early Pancreatic Fibrotic Responses and Suppresses the Profibrotic Cytokine Thrombospondin-1
}

\author{
John C. Schmitz, ${ }^{*}$ Petr Protiva, ${ }^{\star \dagger}$ Arijeet K. Gattu, ${ }^{\dagger}$ \\ Teruo Utsumi, ${ }^{, \dagger}$ Yasuko Iwakiri, ${ }^{\dagger}$ \\ Antonio G. Neto, ${ }^{\dagger}$ Margo Quinn, ${ }^{\ddagger}$ \\ Mona L. Cornwell, ${ }^{\ddagger}$ Philip Fitchev, ${ }^{\ddagger}$ \\ Aurelia Lugea, ${ }^{\text {§ा }}$ Susan E. Crawford, ${ }^{\ddagger}$ and \\ Chuhan Chung ${ }^{\star \dagger}$ \\ From the Section of Digestive Diseases, "VA Connecticut \\ Healthcare System, New Haven, Connecticut; the Department of \\ Medicine, ${ }^{\dagger}$ Yale University School of Medicine, New Haven, \\ Connecticut; the Department of Surgery, ${ }^{\ddagger}$ NorthShore Research \\ Institute, University of Chicago Pritzker School of Medicine, \\ Chicago, Illinois; the Pancreatic Research Group, ${ }^{\circledR}$ Department of \\ Veterans Affairs Greater Los Angeles, University of California Los \\ Angeles, California; and the Southern California Research Center \\ for Alcoholic Liver and Pancreatic Diseases, "Los Angeles, \\ California
}

Pigment epithelium-derived factor (PEDF) is important for maintaining the normal extracellular matrix. We hypothesized that the initiation of pancreatic fibrosis is dependent on the loss of PEDF. Pancreatic PEDF expression was assessed in wild-type mice fed either a control or ethanol diet using an intragastric feeding model. Pancreatitis responses were elicited with either a single episode or a repetitive ceruleininduced ( $50 \mu \mathrm{g} / \mathrm{kg}$, 6 hourly i.p. injections) protocol in wild-type and PEDF-null mice. Quantitative realtime PCR and immunoblotting were performed to assess fibrogenic responses. In wild-type animals, PEDF expression increased with pancreatitis and was more pronounced in mice fed ethanol. Compared with wild-type mice, $\alpha$-smooth muscle actin staining and expression levels of fibrogenic markers ( $\mathrm{eg}$, transforming growth factor- $\beta 1$, platelet-derived growth factor, collagen $I$, and thrombospondin-1) were higher in PEDF-null mice at baseline. Sirius red staining revealed more fibrosis in PEDF-null versus wildtype pancreas 1 week after pancreatitis. Differences in tissue fibrosis resolved with longer recovery periods. PEDF overexpression suppressed thrombospondin-1 levels in vitro. Ethanol feeding and experi- mental pancreatitis increased PEDF expression in wildtype mice. PEDF-null mice, however, demonstrated enhanced early fibrotic responses compared with wildtype mice with pancreatitis. These findings indicate that PEDF acts as a compensatory antifibrotic cytokine in pancreatitis. (Am J Pathol 2011, 179:2990-2999; DOI: 10.1016/j.ajpath.2011.08.009)

Chronic pancreatitis represents a wound-healing response, whereby fibrous scar tissue replaces normal pancreatic tissue. ${ }^{1}$ The major clinical manifestations of chronic pancreatitis include chronic pain and a progressive decline in exocrine/endocrine function, which result from this deposition of fibrous tissue. The principal determinant of pancreatic fibrosis is the pancreatic stellate cell (PSC). In response to injury, PSCs become activated, acquire a profibrotic phenotype, degrade the normal matrix, and secrete matrix proteins necessary for scar formation, including fibrillar collagen. ${ }^{2,3}$ Similar to hepatic stellate cells, the signals that lead to PSC activation and proliferation have been extensively described. ${ }^{2}$ Little is known, however, about the regulators that maintain PSC in a quiescent state.

One endogenous regulator of fibroblast quiescence is pigment epithelium-derived factor (PEDF). ${ }^{4-10}$ Best studied as a potent inhibitor of angiogenesis, PEDF is a 46$\mathrm{kDa}$ secretory protein with a serpin structure lacking protease inhibitory activity. ${ }^{11-14}$ PEDF also binds with high affinity to matrix components, such as collagen I. $^{15}$ PEDF's inhibitory effect on mesenchymal cell proliferation in vitro was because of its ability to induce cell cycle arrest. $^{4,5,16}$ In fibroblasts, PEDF inhibits S-phase entry, whereas its absence allows for DNA replication and cel-

Supported by the Veterans Affairs CDA Award (C.C.), R21AA015781 (A.L.), R01CA64239-10S1 (S.E.C.), Veterans Affairs Merit Award, and the National Pancreas Foundation grant (C.C.).

Accepted for publication August 10, 2011

Supplemental material for this article can be found at http://ajp. amjpathol.org or at doi: 10.1016/j.ajpath.2011.08.009.

Address reprint requests to Chuhan Chung, M.D., Section of Digestive Diseases, VA CT Research, Yale University School of Medicine, GI Research Bldg 4, West Haven, CT 06516. E-mail: chuhan.chung@yale.edu. 
lular proliferation. ${ }^{5,17}$ Genetic deletion of PEDF in mice supports this role. PEDF-null mice display stromal expansion in several organs, including the pancreas and liver. ${ }^{6,9}$ Thus, endogenous PEDF restrains the proliferation of cells that are relevant to fibrogenic responses in the normal pancreatic matrix.

The loss of PEDF in genetic and disease models reflects the findings reported from these cellular studies. We previously reported that $\alpha$-smooth muscle actin expression occurs in the perisinusoidal space, the site of stellate cells, in livers of PEDF-null mice. ${ }^{9}$ Furthermore, long-term ethanol feeding in mice and humans resulted in the loss of PEDF in rodent and human livers and in hepatic stellate cell activation. ${ }^{9}$ Decreased PEDF levels also occurred in cirrhotic human and rodent livers compared with normal specimens, suggesting that its loss is permissive for liver fibrosis. ${ }^{10}$ Moreover, PEDF delivery in two models of cirrhosis markedly reduced stellate cell activation and liver fibrosis through induction of antifibrogenic transcription factors, such as peroxisome proliferator-activated receptor- $\gamma$, and suppression of pro-inflammatory cytokines, such as monocyte chemotactic protein-1. ${ }^{10}$ The antifibrogenic properties of PEDF have also been demonstrated in other organs, including the kidney, heart, and eye. . $^{7,18,19}$ PEDF delivery ameliorated kidney fibrosis and proteinuria by suppressing transforming growth factor (TGF)- $\beta 1$ expression in diabetic rats. ${ }^{8} \mathrm{Sim}$ ilarly, in a model of acute myocardial infarction, PEDF infusion reduced cardiac fibrosis through down-regulation of TGF- $\beta 1$ and fibrillar collagen expression, thereby improving left ventricular ejection. ${ }^{18}$ These in vivo studies support earlier cellular studies that identified PEDF as a quiescence factor for fibroblasts.

In contrast to the reported antifibrogenic effects of PEDF in the liver and other organs, a recent report ${ }^{20}$ suggested that PEDF could have profibrogenic effects in the pancreas. To determine PEDF's role in pancreatic fibrogenesis, we investigated PEDF expression and fibrotic responses after cerulein-induced pancreatitis in wild-type mice and in mice with genetic deletion of PEDF. We then explored whether the absence of PEDF affects matrix proteins known to function in angiogenesis and tissue repair processes, such as thrombospondin-1 (TSP-1). TSP-1 is a multifunctional matrix protein expressed at sites of wound repair ${ }^{21,22}$ that acts as a profibrogenic cytokine by activating latent TGF- $\beta 1$ and regulating fibrillar collagen expression. ${ }^{22-24}$ TSP-1 blockade has been shown to reduce TGF- $\beta 1$ signaling and tissue fibrosis. ${ }^{25-28}$ TSP- 1 expression also markedly increased after experimental acute pancreatitis. ${ }^{22}$ Thus, it would be expected that PEDF and TSP-1 would be reciprocally regulated if PEDF functions to inhibit fibrosis in the pancreas.

\section{Materials and Methods}

\section{Animals}

To determine the effects of ethanol feeding and ceruleininduced pancreatitis on pancreatic levels of PEDF, archival pancreatic tissue was obtained from the University of
Southern California-University of California, Los Angeles, Alcohol Research Center through a collaborator (A.L.). In this protocol, mice were fed an ethanol-containing or control diet for 5 weeks using the intragastric TsukamotoFrench model. ${ }^{29}$ After 10 days on the diets, mice were subjected to one episode or five repetitive episodes (50 $\mu \mathrm{g} / \mathrm{kg}, 6$ hourly i.p. injections) of cerulein-induced pancreatitis with an interval of 3 days. Mice given a single episode of pancreatitis were sacrificed 1 hour after the last cerulein injection. Mice on the repetitive protocol were sacrificed 4 days after the last course of pancreatitis, and pancreatic tissue was collected for histological analysis.

To assess the role of PEDF in pancreatic fibrosis, PEDF-deficient mice and age-matched wild-type littermates were used. ${ }^{6}$ Offspring were genotyped using a commercially available PCR kit (Sigma, St. Louis, MO). PEDF-null mice were viable and produced normal-sized litters. Animals received cerulein $(50 \mu \mathrm{g} / \mathrm{kg})$ i.p. hourly for 7 consecutive hours on an every other day basis for 2 weeks. Animals $(n=6)$ were sacrificed at 1,4 , and 12 weeks after the completion of the pancreatitis protocol. This protocol previously resulted in fibrotic changes without obvious exocrine insufficiency. ${ }^{30}$ Because of the severity of tissue injury 1 week after this protocol, only the tissue adjacent to the spleen was obtained. A separate set of null and wild-type animals $(n=3)$ received an equivalent volume of saline i.p. and served as controls. All procedures were approved by the Institutional Animal Care and Use Committee of VA Connecticut and Greater Los Angeles VA Healthcare System.

\section{Histological Findings}

Tissue samples were stained with H\&E and trichrome, as previously described. ${ }^{9}$ Immunohistochemistry $(\mathrm{IHC})$ was performed using a streptavidin-biotin complex immuneperoxidase technique. Sections were deparaffinized, treated to inhibit endogenous peroxidase, and subjected to antigen retrieval. Slides were washed in Tris-buffered saline and then primary antibodies were added at optimal dilutions: PEDF (1:100), ${ }^{6}$ TSP-1 Ab-11 (1:100; NeoMarkers, Fremont, CA), and $\alpha$-smooth muscle actin (1:50; Wako, Richmond, VA). After incubation with primary antibody, sections were washed and then incubated with biotinylated antimouse antiserum. Streptavidin complexed with horseradish peroxidase was added, and labeling was detected using diaminobenzidine. Two experienced pathologists (S.E.C. and A.G.N.) performed all histological evaluations in a blinded manner.

To assess pancreatic collagen content, sections were stained with Sirius red. Sections were pretreated to remove paraffin and stained in $0.1 \%$ Sirius red solution in saturated picric acid for 1 hour. Slides were washed in $0.09 \mathrm{~N}$ acetic acid, dehydrated in 100\% ethanol, cleared in xylene, and mounted. Images were captured with exposures manually set at equal times for all sections using a Leica DM4000 B microscope. Additional images were captured on a polarizing microscope. Nonoverlapping images from each pancreas were acquired using the $\times 10$ objective. Quantification of IHC immunolabeling and 
collagen accumulation was evaluated by morphometric analysis using $\mathrm{NIH}$ ImageJ software (Bethesda, MD). ${ }^{31}$ Results were normalized to control values and expressed as a percentage to baseline control values.

\section{Cell Culture}

The human pancreatic cancer cell lines Panc- 1 and Mi$\mathrm{aPaCa}$ were maintained according to American Type Culture Collection guidelines. To obtain conditioned medium (CM), cells were grown to $80 \%$ confluence, washed twice with serum-free media, and then incubated with serum-free media overnight. To obtain lysates, cells were scraped with radioimmunoprecipitation assay buffer, incubated on ice, and centrifuged at $10,000 \times g$ for 10 minutes. $\mathrm{CM}$ was obtained after 18 to 20 hours and concentrated approximately 40-fold using Amicon Ultra centrifugal filters (Millipore, Billerica, MA) with a 10-kDa cutoff.

\section{Quantitative Real-Time PCR}

Total RNA was extracted from pancreatic samples using TRIzol reagent (Invitrogen, Carlsbad, CA). Total RNA, $1 \mu \mathrm{g}$, was reverse transcribed to cDNA using SuperScript II RNaseH reverse transcriptase and $50 \mathrm{ng}$ of random hexamers (Invitrogen). RNA purity was assessed using a BioAnalyzer 2100 (Agilent). Quantitative real-time PCR was performed on a TaqMan ABI 7000 detection system using TaqMan Universal Mastermix (Applied Biosystems, Foster City, CA). Primers for PEDF, TGF- $\beta 1$, platelet-derived growth factor (PDGF), collagen subtypes (I $\alpha 1,4 \alpha 2,5 \alpha 1)$, fibroblast growth factor 2, TSP-1, TSP-2, matrix metalloproteinase (MMP)-2, and MMP-9 were obtained from Applied Biosystems. The mRNA levels of target genes were normalized to $18 \mathrm{~S}$ ribosomal RNA levels.

\section{PEDF Transfection of PSCs and Pancreatic Cancer Cells}

The human PEDF gene or its empty vector, pCEP4 (Invitrogen), was transfected into Panc-1 cells using Lipofectamine 2000. To knock down PEDF, commercial smallinterfering RNA (siRNA) constructs (Ambion, Foster City, $\mathrm{CA}$ ) were used according to the manufacturer's instructions. After 6 hours, transfection medium was removed and fresh medium was added. After an additional 48 hours, the medium was changed to serum-free medium for 24 hours. CM and cell lysates were obtained as previously described.

\section{Immunoblotting}

Immunoblotting was performed as previously described. ${ }^{9}$ Protein content was determined by the Bradford assay. Pancreatic lysates (30 $\mu \mathrm{g}$ of total protein) were run under denaturing conditions on a gradient gel (Bio-Rad, Hercules, CA) and then transferred to polyvinylidene difluoride membranes. After blocking in a $5 \%$ milk solution, membranes were incubated overnight with antibodies. The primary antibodies used were as follows:
PEDF (1:1000; Chemicon, Temecula, CA), TSP-1 (1:1000; NeoMarkers Ab-11), MMP-2 and MMP-9 (1:1000; Chemicon), TGF- $\beta 1$ (1:1000; Cell Signaling, Danvers, MA), phospho-SMAD2 (serine 465/467, 1:1000; Cell Signaling), collagen I $\alpha 1$ (1:200; Santa Cruz, Santa Cruz, CA), vascular endothelial growth factor (1:200; AbCam, Cambridge, MA), anti-thyroglobulin (1:500; Cayman, Ann Arbor, MI), $\beta$-actin (1:2500; Sigma), and $\alpha$-tubulin (1:5000; Calbiochem, La Jolla, CA). After washing in Tris-buffered saline and $0.05 \%$ Tween, the primary antibody was labeled using a peroxidase-conjugated antibody specific for the primary antibody species. For phospho-SMAD2 detection, lysates were freshly harvested with commercial phosphatase inhibitors (Cocktail 2 and 3; Sigma). Samples were resolved on a gradient gel and transferred to nitrocellulose membranes. Equivalence of loading was confirmed using $\beta$-actin or Coomassie stain of CM. Densitometry was performed as previously described using $\mathrm{NIH}$ ImageJ software. ${ }^{9}$

\section{Statistical Analysis}

$P$ values were calculated, assuming equal sample variance, using two-tailed Student's $t$-tests on Prism software (GraphPad Software Inc., La Jolla, CA), with $P<0.05$ deemed statistically significant. Analysis of more than two groups was performed using one-way analysis of variance, with $P<0.05$ deemed significant. The Tukey's multiple comparison test was used to assess significance between groups. Values were stated as mean $\pm \mathrm{SE}$.

\section{Results}

\section{PEDF Expression Is Induced in the Pancreas of Wild-Type Mice Fed Ethanol and Subjected to Cerulein-Induced Pancreatitis}

The intensity of PEDF immunolabeling in wild-type mice increased with the severity of cerulein-induced pancreatitis and was more pronounced in animals fed ethanol (Figure 1, A-F). In mice fed a control diet, PEDF labeling intensity was prominent in islets (Figure $1 \mathrm{~A}$ ) and parenchymal staining was faint, patchy, or not apparent in large regions. An ethanol diet increased PEDF labeling in a patchy distribution, with some acinar cells demonstrating diffuse labeling and adjacent cells relatively devoid of PEDF staining (Figure 1B). A single episode of ceruleininduced pancreatitis led to diffuse labeling in the exocrine pancreas (Figure 1C) that was accentuated by ethanol feeding (Figure 1D). With the repetitive pancreatitis protocol, PEDF staining intensified and became more diffuse (Figure 1E). This was further augmented in ethanol-fed animals, in which staining was most robust in areas of stromal expansion with ductal regeneration (Figure 1F). Densitometry of PEDF immunolabeling revealed that PEDF increased with both ethanol exposure and cerulein-induced pancreatitis and was $>50 \%$ higher than controls when repetitive cerulein was combined with ethanol feeding $(P<0.001$, Figure $1 \mathrm{G})$. Quantification of pancreatic tissue from mice with combined ethanol and 

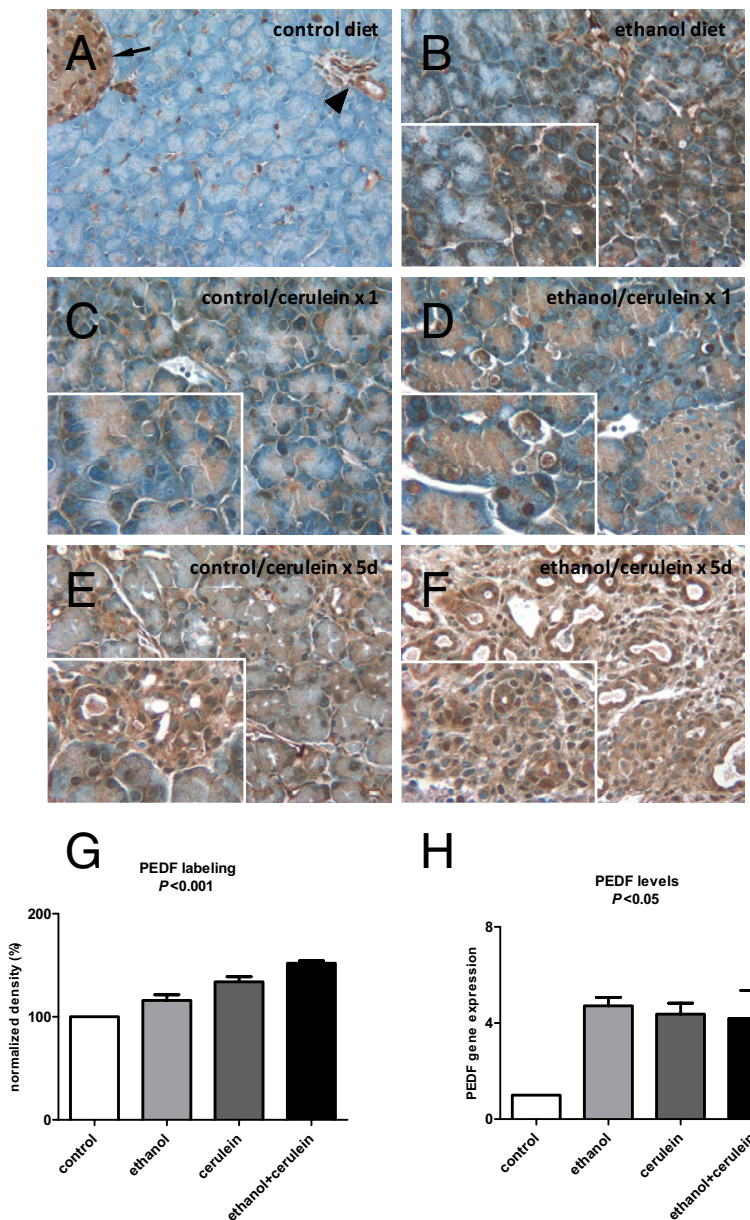

$\mathrm{H}$

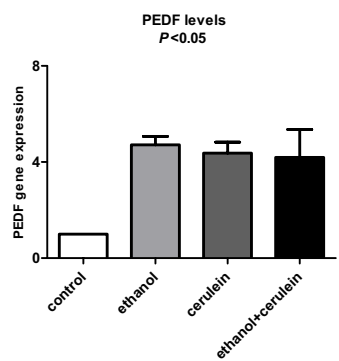

Figure 1. Cerulein-induced pancreatitis increases PEDF levels in wildtype mice and is accentuated with ethanol feeding. A: PEDF labeling (brown stain) was faint in mice fed a control diet, except in islets (arrow) and ducts/vessels (arrowhead). B: In mice fed ethanol for 5 weeks, moderate PEDF staining was apparent in acinar cells in a patchy distribution with strong staining adjacent to regions with little staining (inset) PEDF labeling was faint after one dose of cerulein (C) but became more prominent in the setting of ethanol feeding (D). A repetitive cerulein protocol led to diffuse PEDF labeling (E) that was pronounced in animals fed ethanol $(\mathbf{F})$, with prominent staining in areas of stromal expansion and ductular proliferation (inset). G: Densitometry of PEDF immunolabeling revealed progressively increasing density with ethanol feeding, repetitive cerulein injection, and repetitive cerulein injection plus ethanol feeding. H: $P E D F$ gene expression increased with ethanol feeding and pancreatitis alone and combined.

cerulein injection, moreover, may have underestimated the extent of staining due to areas occupied by robust ductular proliferation (Figure 1F), a histological finding that was most pronounced in this group. PEDF mRNA levels from saline-injected controls and animals subjected to the repetitive pancreatitis protocol mirrored these histological findings $(P<0.05$, Figure $1 \mathrm{H})$. PEDF, therefore, increases with pancreatitis and its intensity increases with ethanol feeding.

\section{Absence of PEDF Promotes Stromal Activation and a Pancreas That Is Primed for Fibrotic Injury}

Because of its direct role in suppressing mesenchymal proliferation, the absence of PEDF is permissive for hepatic stellate cell activation and its delivery can suppress stellate cell activation and tissue fibrosis. ${ }^{5,9,10}$ Similarly, the pancreas of PEDF-null mice displayed baseline enhanced $\alpha$-smooth muscle actin expression, consistent with PSC activation (Figure 2A). The pancreatic gene expression profile of PEDF-null mice mirrored these findings. Expression levels of fibrogenic markers, such as TGF- $\beta 1(P<0.001)$, PDGF $(P<$ $0.01)$, collagen I $\alpha 1(P<0.05)$, and fibroblast growth factor $2(P<0.05)$, were all significantly higher at baseline in the PEDF-null mice (Figure 2B). MMP-2 and MMP-7 (data not shown) expression levels were significantly higher in PEDF-null mice compared with wildtype mice. Immunoblots of fibrogenic markers (Figure 2C) and their corresponding densitometry results (Figure 2D) corroborated these findings. Levels of the cleaved active form of TGF- $\beta 1$, its transcriptional mediator, phospho-SMAD2 (serine 465/467), and the in vivo TGF- $\beta 1$ activator, TSP-1, were significantly higher in PEDF-null mice. MMP-2 levels reflected the relative abundance of gene expression and are consistent with previously published data on PEDF's inhibitory effects on MMP-2 levels and activity in animal models of myocardial infarction. ${ }^{18}$ MMP-9 protein levels, however, were lower in PEDF-null mice, indicating the differential regulation of MMP-2 and MMP-9. ${ }^{32,33}$ Despite these differences in profibrogenic cytokines, peri-acinar and lobular fibrosis was not visibly greater in PEDF-null mice than in wild-type mice, as determined by Sirius red staining in saline-injected mice (data not shown). This is also reflected in comparable protein levels of collagen I $\alpha 1$ at baseline, despite elevated gene expression of multiple collagen species (Figure 2B; see also Supplemental Figure S1 at http://ajp.amjpathol. org). These results indicate that the absence of PEDF at baseline does not result in histological tissue fibrosis but may predispose the pancreas to additional injury.

\section{Cerulein-Induced Pancreatitis in PEDF-Null Mice Results in Enhanced Early Fibrosis}

A 2-week cerulein injection protocol to induce early fibrotic changes was used to ascertain whether the absence of PEDF could generate pathological tissue fibrosis. This protocol induces fibrotic changes but has not resulted in overt exocrine or endocrine insufficiency. ${ }^{30}$ Weight changes were tracked for up to 4 weeks after cerulein injection as a potential marker of pancreatic insufficiency. Wild-type animals recovered weight up to their baseline at approximately 2 weeks and gained weight above baseline levels thereafter (Figure 3A). PEDF-null mice, however, were unable to recover weight to baseline levels up to 4 weeks after the last cerulein injection (Figure 3A; percentage weight loss in PEDF-null versus wild-type mice; $P<$ 0.01). Trichrome staining of pancreas obtained after a 1-week recovery period confirmed abundant periductular collagen deposition and mild changes in the 


\section{A}
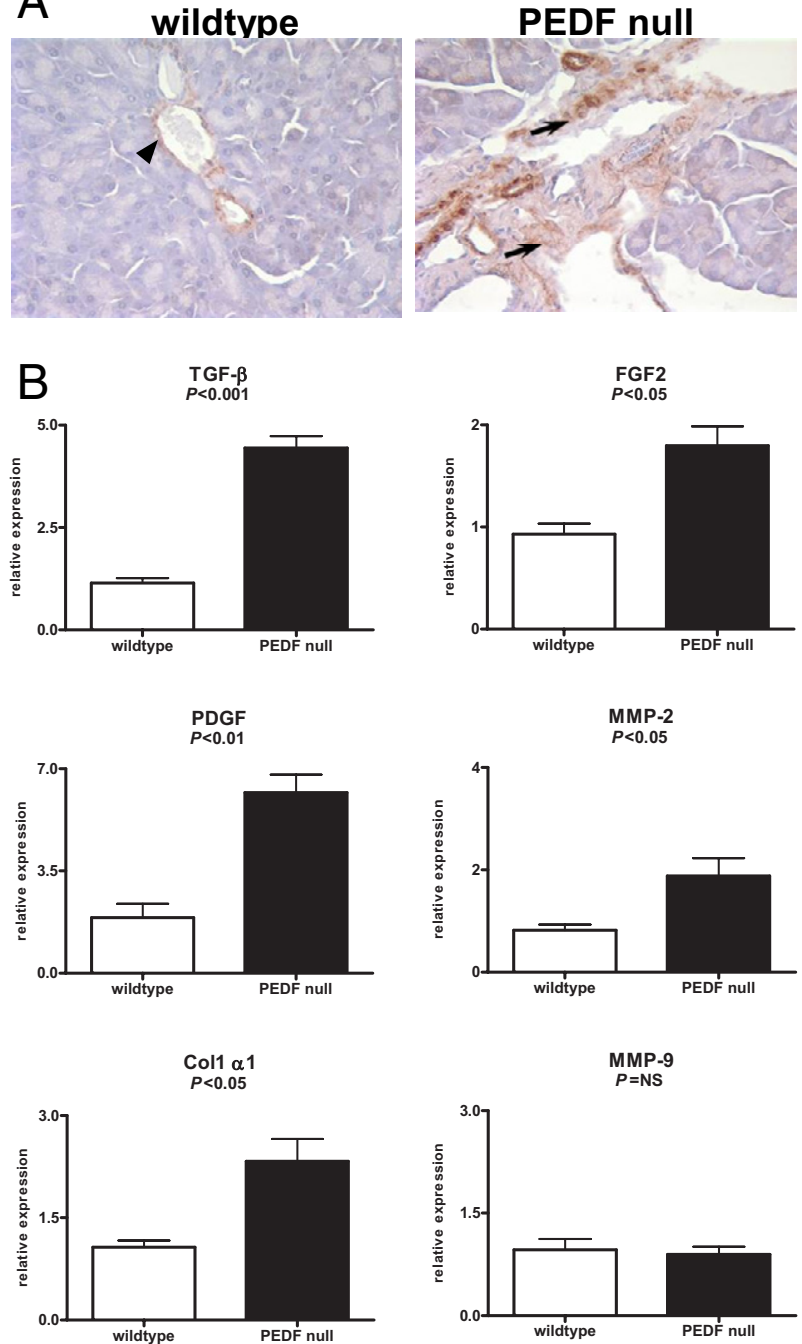

Figure 2. The PEDF-null pancreas at baseline demonstrates $\alpha$-smooth muscle actin ( $\alpha$-SMA) staining and a cytokine profile that predisposes to tissue fibrosis. A: The pancreas of wild-type (left) and PEDF-null (right) mice revealed $\alpha$-SMA staining in wild-type blood vessels (arrowhead) and ducts, whereas PEDF-null mice demonstrated prominent $\alpha$-SMA positivity (arrows) at baseline, indicating the presence of activated PSCs. B: mRNA expression levels of profibrogenic factors, such as TGF- $\beta 1$, PDGF, MMP-2, fibroblast growth factor (FGF) 2 , and collagen (Col) I, were significantly increased in the PEDF-null pancreas compared with controls. C: Immunoblots for TGF- $\beta 1$ demonstrated increased active to total TGF- $\beta 1$ levels. Consistent with this finding were elevated levels of phospho-SMAD2 and TSP-1 in the PEDF-null pancreas. Greater MMP-2 levels in the PEDF-nul pancreas reflected findings seen on real-time PCR, whereas Col I $\alpha 1$ levels were not different between groups. D: Corresponding densitometry of immunoblots in $\mathbf{C}$.

peri-acinar region (Figure 3B). PEDF-null mice, however, displayed more robust fibrotic changes; this was most apparent around ductular and acinar structures (Figure 3B). Images captured with a polarizing microscope and Sirius red stains were consistent with this enhanced fibrosis (Figure 3C). Quantification of Sirius red stains demonstrated $44 \%$ greater fibrosis in PEDFnull compared with wild-type mice (Figure 3D, $P<$ $0.01)$. Thus, the absence of PEDF promotes enhanced pancreatic fibrosis after a 2-week protocol of repetitive cerulein-induced pancreatitis and 1-week recovery.

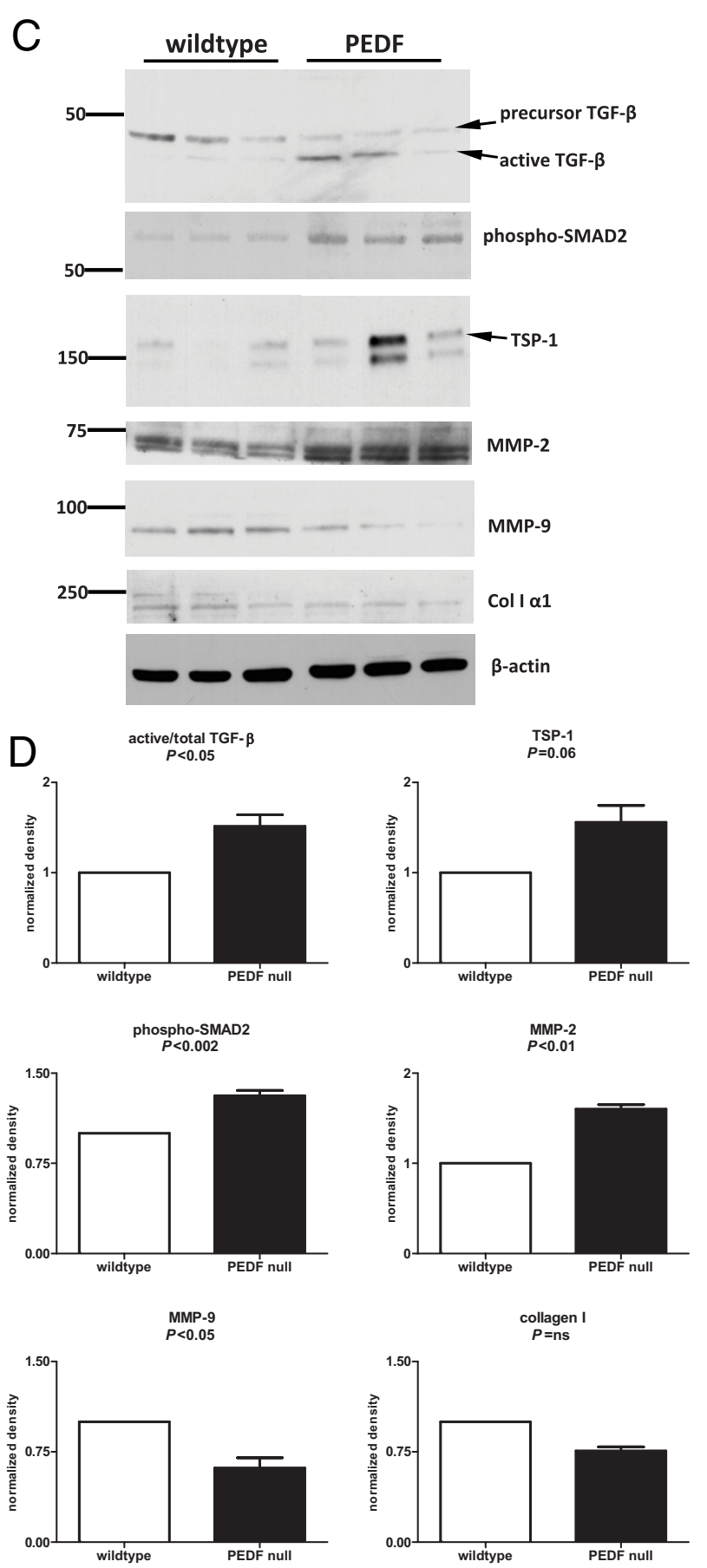

Fibrosis in PEDF-Null Mice Resolves after 4-Week Recovery and Is Accompanied by a Blunted Fibrogenic Response

Because of the enhanced fibrosis seen in the PEDF-null pancreas 1 week after cerulein injection, we anticipated further injury in animals with prolonged recovery periods. On the contrary, substantial resolution of fibrosis occurred in both groups of animals, with remnant fibrous deposition similar in both groups of animals (Figure 4A). In PEDF-null mice, trichrome 


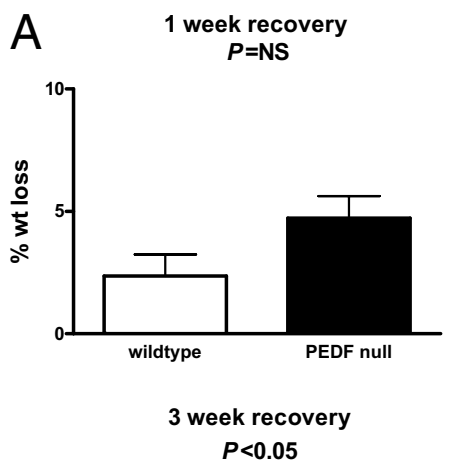

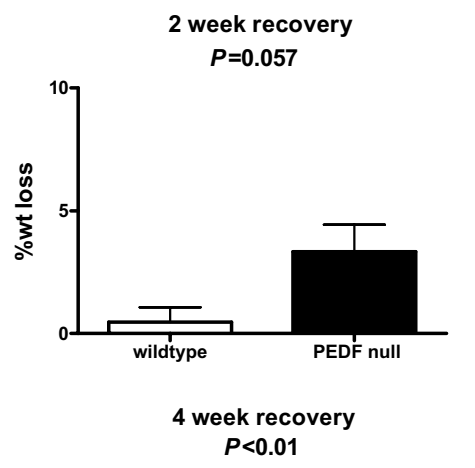

\section{B wildtype}
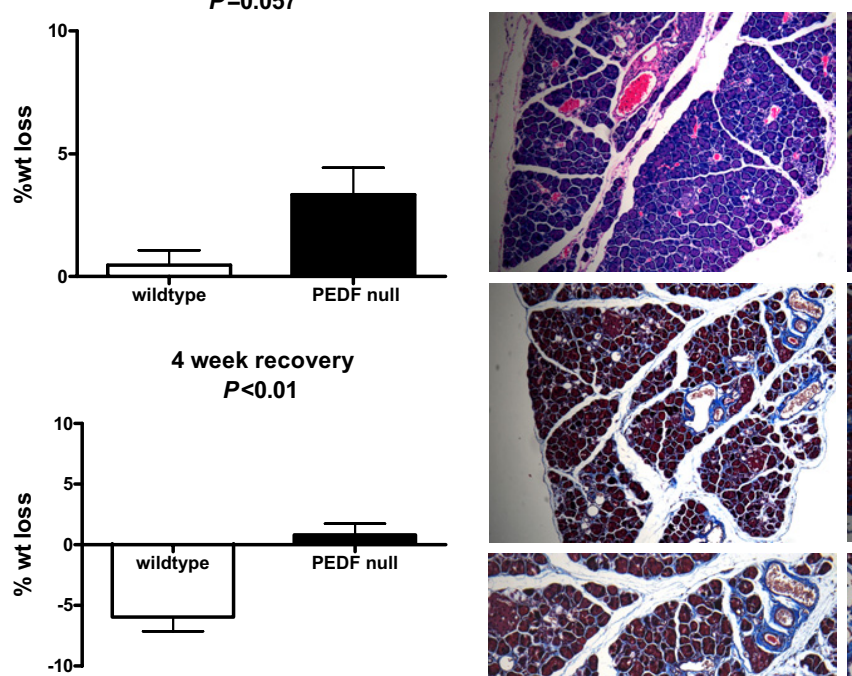

PEDF null
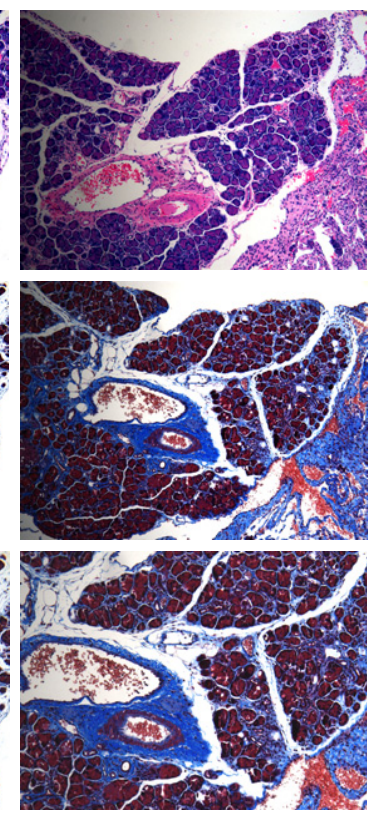

\section{C wildtype}
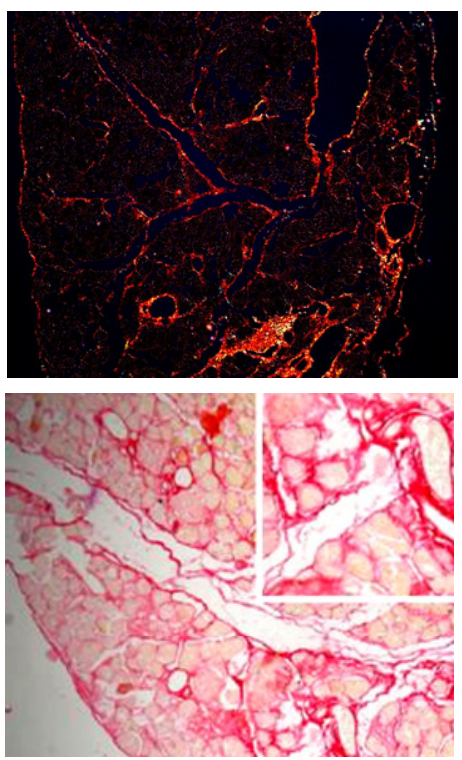

\section{PEDF null}
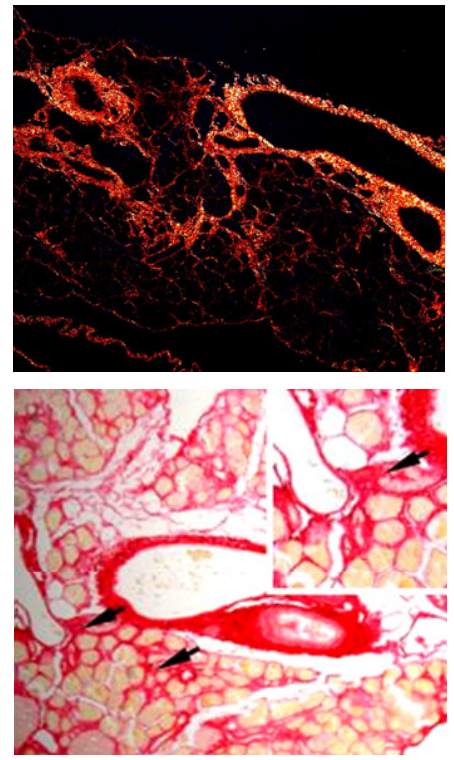

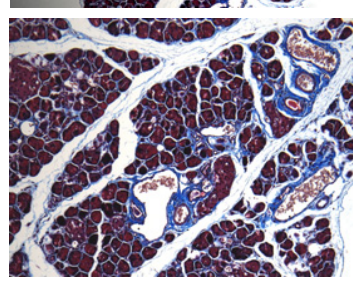

D

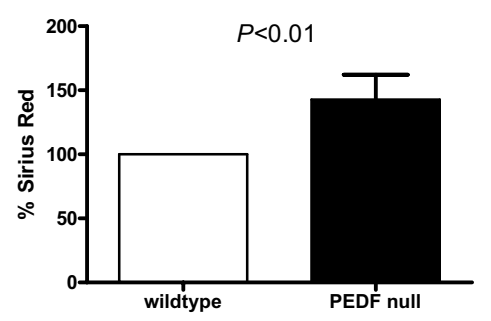

Figure 3. PEDF-null mice fail to recover body weight and demonstrate increased fibrous deposition after experimental pancreatitis. A: PEDF-null mice demonstrated significant weight loss that persisted up to 4 weeks after the cessation of experimental pancreatitis. B: H\&E (top) and trichrome (middle and bottom) stains from mice 1 week after pancreatitis demonstrate stromal expansion and increased fibrosis in the PEDF-null pancreas. Images captured with polarized light $(\mathbf{C})$ and Sirius red stains showing increased fibrosis deposition around acini (arrows) with quantification (D) corroborate the increased fibrosis seen in PEDF-null compared with wildtype mice. staining regressed almost completely (Figure 4A), except in areas immediately surrounding pancreatic ducts and vessels. Trichrome staining persisted in wild-type mice adjacent to acini and lobules (Figure 4A), but Sirius red stains and quantification did not reveal significant differences. To ascertain why the degree of tissue fibrosis appeared to reverse with longer recovery periods, we compared the profibrogenic cytokine profile at baseline with that found with extended recovery. As expected, TGF- $\beta 1$ expression increased in both groups after pancreatitis (Figure 4B). However, TGF- $\beta 1$ expression was higher in wild-type compared with PEDF-null mice $(P<0.01)$. Other fibrogenic markers, including PDGF, fibroblast growth factor 2, and collagen I, demonstrated increased expression with pancreatitis in the wild-type pan- creas, whereas those from PEDF-null mice demonstrated a muted fibrogenic response after a 4-week recovery (Figure $4 \mathrm{~B})$. Thus, profibrogenic cytokines in PEDF-null mice are elevated at baseline, but their expression is blunted with prolonged recovery after cerulein-induced pancreatitis.

\section{TSP-1 Demonstrates Low Levels of Expression} in the Wild-Type Pancreas and Increases with the Absence of PEDF in Vivo

To ascertain if the early profibrogenic effects seen in PEDF-null mice were related to the regulation of antiangiogenic proteins implicated in tissue fibrosis, TSP-1 
A
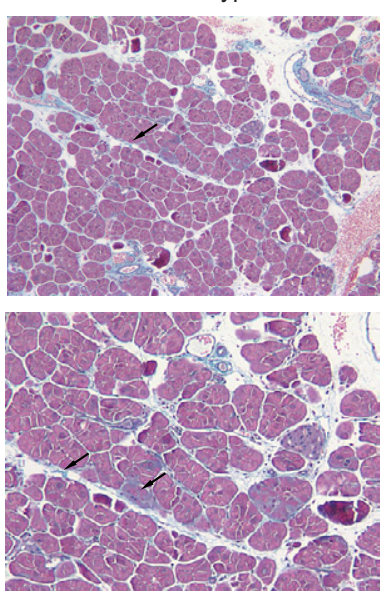

B
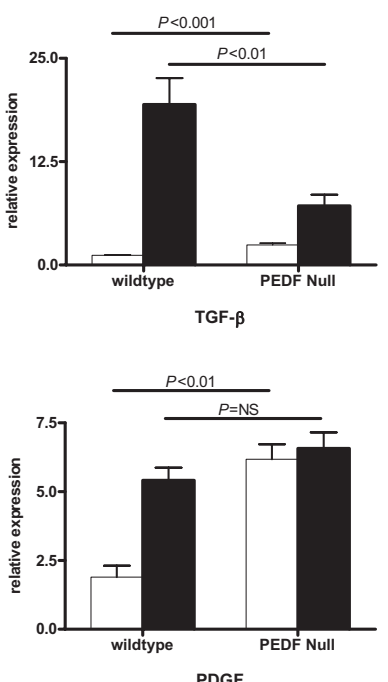

PEDF null
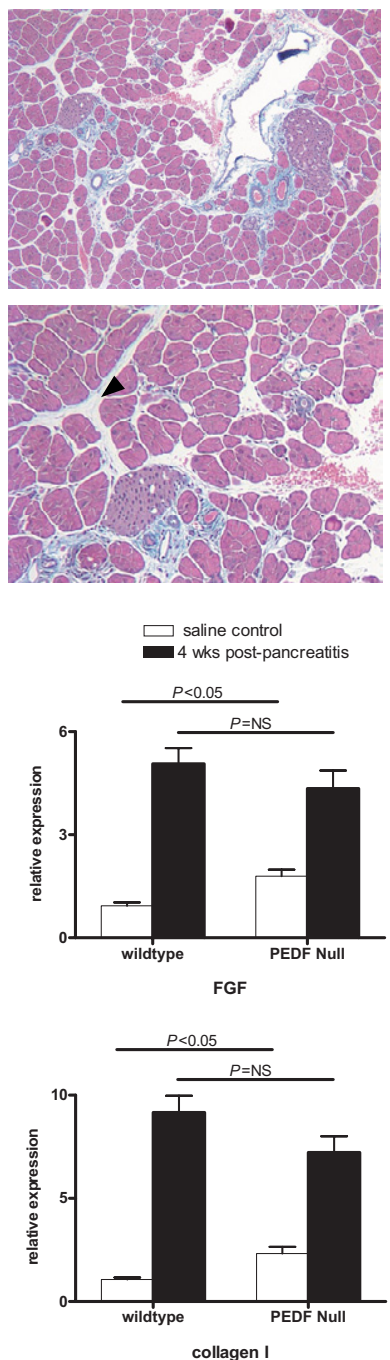

Figure 4. Four weeks after pancreatitis, PEDF-null mice demonstrate resolution of injury and a blunted fibrogenic cytokine profile. A: Trichrome stains demonstrate scant collagen deposition around lobules in wild-type pancreas (left, arrows), whereas PEDF-null mice showed resolution of perilobular fibrosis (right, arrowhead). B: Expression levels of fibrogenic markers show a predicted increase in wild-type mice. At 4 weeks after pancreatitis, PEDF-null mice reveal a fibrogenic cytokine profile that is similar to wildtype animals.

levels were evaluated because of its known actions as an activator of TGF- $\beta 1 .^{23}$ TSP-1 gene expression is low in the native pancreas, but histological staining for TSP-1 in the normal pancreas is not well characterized. ${ }^{22}$ Given its induction and role in wound repair, ${ }^{21}$ we anticipated that TSP-1 expression would be regulated by PEDF in vivo. Saline-injected wild-type mice demonstrated minimal staining for TSP-1 in the pancreatic parenchyma (Figure 5A). PEDF-null mice, in contrast, displayed distinct TSP-1 immunolabeling in areas adjacent to ducts and blood vessels (Figure 5A). Differences in TSP-1 labeling became less apparent in animals 4 weeks after pancreatitis because wild-type animals displayed more prominent TSP-1 staining. Unlike the earlier time point, the wild-type pancreas displayed TSP-1 staining in areas of tissue repair, which was also evident in PEDF-null mice (Figure

5A). Real-time PCR results reflected these findings with fourfold higher TSP-1 expression levels in saline-injected PEDF-null mice compared with wild-type controls (Figure 5B, $P<0.05$ ). Four weeks after pancreatitis, TSP-1 levels were elevated threefold in wild-type animals, whereas TSP-1 levels remained unchanged in PEDF-null mice (Figure 5B). The absence of PEDF, therefore, is associated with enhanced TSP-1 expression in the pancreas and suggests a direct signaling mechanism between these two proteins to modify the stromal cellular response to an inflammatory stimulus.

\section{PEDF Levels Modulate the Expression of the Fibrogenic Regulator TSP-1 in Vitro}

Unlike our findings in the PEDF-null mice, previous reports $^{34,35}$ had indicated that PEDF and TSP-1 are coordinately regulated. To investigate a potential suppressive effect of PEDF on TSP-1, PEDF was overexpressed in Panc- 1 cells (a pancreatic cancer cell line with relatively low PEDF levels). This resulted in a striking suppression of TSP-1 (Figure 6) in cell lysates and in the CM (see Supplemental Figure S2A at http://ajp.amjpathol.org). Molecular knockdown of PEDF-overexpressing Panc-1 cells using siRNA reversed this process, restoring TSP-1 to baseline levels (Figure 6). This effect appears to be cell type specific because PEDF siRNA did not alter TSP-1 levels in pancreatic cancer cells with high endogenous PEDF levels (see Supplemental Figure S2B at http:// ajp.amjpathol.org). Thus, we have shown that PEDF reciprocally modulates TSP-1 both in vitro and in vivo.

A
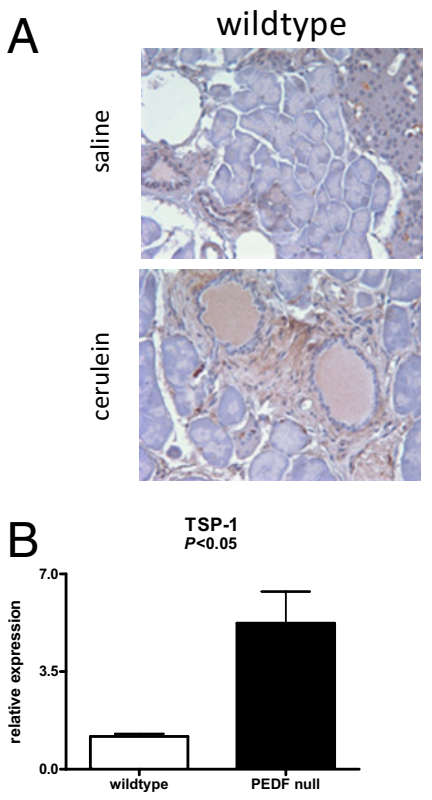

Figure 5. PEDF and TSP-1 are reciprocally regulated in the baseline pancreas. A:Immunolabeling for TSP-1 demonstrated increased localization around vessels and ducts but not lobules in PEDF-null mice compared with wild-type animals (top). After pancreatitis induction and 4-week recovery, both wild-type and PEDF-null pancreas displayed increased TSP-1 labeling (bottom). B: TSP-1 expression levels reflected histological changes with increased TSP-1 levels at baseline in PEDF-null compared with wild-type pancreas $(P<0.05)$ but no differences after pancreatitis. 

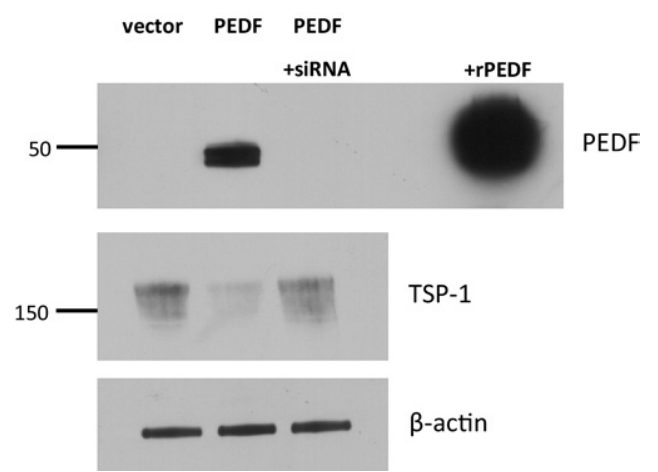

Figure 6. PEDF overexpression suppresses TSP-1 levels; PEDF siRNA restores TSP-1. Panc-1 cells were transfected with either pCEP4 vector alone or pCEP4-PEDF vector. Top: PEDF in cell lysates was not seen with vector alone but only with PEDF transfection. PEDF siRNA abolished PEDF expression. Middle: Detection of TSP-1 in cell lysates from corresponding conditions revealed that PEDF can suppress TSP-1, whereas knockdown of PEDF can restore TSP-1 levels. Bottom: $\beta$-Actin labeling demonstrates equal loading. Images are representative of three separate experiments.

\section{Discussion}

Chronic pancreatitis represents the wound-healing response to repetitive injury, with PSC activation and the deposition of fibrillar collagen composing key steps in this process. A multitude of factors are important for PSC activation. ${ }^{2}$ Among these are factors such as TGF- $\beta 1$ and PDGF that are known to be fibrogenic in other organs, such as the liver. ${ }^{36}$ The factors that serve as endogenous and negative modulators of fibroblast activation, however, are less well understood. ${ }^{16}$ Recent studies ${ }^{8-10,18,19}$ have shown that PEDF negatively regulates stromal cell activation in vivo and that systemic PEDF delivery could ameliorate fibrosis in the kidney, liver, heart, and eye. Consistent with these observations, we found that PEDFnull mice at baseline were primed for pancreatic injury; when subjected to a 2-week course of pancreatitis, these mice exhibited more marked fibrosis at the 1-week recovery period. In addition, impaired weight gain suggested that systemic recovery was compromised. With a longer recovery time of 4 weeks, PEDF-null and wild-type mice demonstrated similar degrees of fibrosis resolution. Thus, the absence of PEDF predisposes to early fibrotic changes in the pancreas but does not inhibit the compensatory mechanisms to resolve tissue fibrosis after pancreatitis.

The combination of increased PEDF staining after pancreatitis in wild-type mice, and the enhanced fibrosis seen in PEDF-null mice, suggests that PEDF functions as a protective factor in experimental pancreatitis and other disease conditions characterized by a fibrotic response. 8,10,18,19 Pancreatic PEDF expression by mRNA and tissue labeling increased with pancreatitis responses. Ethanol feeding accentuated PEDF labeling, which was most robust in regions of stromal expansion and ductular proliferation. This staining pattern was consistent with a recent study ${ }^{20}$ that showed increased PEDF staining in fibrotic areas associated with pancreatic cancer. This study concluded that PEDF acts as a profibrogenic factor in pancreatic cancer. ${ }^{20}$ However, in pancreatitis responses, the early fibrosis seen in PEDF-null mice suggests that PEDF serves as a counterregulatory mechanism in pancreatitis. This function is also consistent with recently published data ${ }^{8,10,18,19}$ on PEDF's antifibrotic role in the kidney, liver, heart, and eye. PEDF overexpression in a transgenic mouse model ameliorated the increase in TGF- $\beta 1$ and inflammatory markers in an eye injury model associated with neovascularization and fibrosis. ${ }^{19}$ Similarly, PEDF delivery ameliorated liver fibrosis by maintaining hepatic stellate cells in a quiescent state and reducing the levels of profibrogenic markers. ${ }^{10}$ Consistent with the antifibrotic effects of PEDF reported in these studies, the absence of PEDF was permissive for increased TGF- $\beta 1$, PDGF, collagen I, and protease expression levels. These data, in conjunction with the findings in the current study, suggest that PEDF expression in pancreatitis represents a counterregulatory measure to inhibit fibrotic responses.

PEDF is best known as an endogenous inhibitor of angiogenesis. However, it also functions as an important regulator of proteins in the stromal compartment. ${ }^{6,10,16}$ Thus, we evaluated whether PEDF could modulate the levels of other anti-angiogenic proteins involved in the tissue repair process, such as TSP-1. TSP-1 is an important modulator of the wound-healing response that is due, in part, to its ability to bind collagen, activate TGF- $\beta 1$, and regulate angiogenesis. ${ }^{21}$ In mice, the absence of PEDF resulted in increased TSP-1 expression and immunolabeling in the basal state. To rule out an indirect effect through other fibrogenic cytokines, this relationship was evaluated with PEDF overexpression experiments in Panc-1 cells, which demonstrated a marked decrease in TSP-1 levels with PEDF expression. Furthermore, PEDF knockdown in PEDF-overexpressing cells with siRNA restored TSP-1 to baseline levels, demonstrating that PEDF modulates TSP-1 expression. PEDF and TSP-1, therefore, can be reciprocally regulated in the mouse pancreas and in some human pancreatic cancer cells. Thus, we have defined a novel regulatory pathway that explains PEDF's ability to inhibit profibrotic processes by suppressing TSP-1.

Because PEDF and TSP-1 share common properties pertaining to their roles as inhibitors of angiogenesis, previous studies had indicated that PEDF and TSP-1 are coordinately regulated. Overexpression of the ras oncogene, for example, suppressed both PEDF and TSP-1. ${ }^{35}$ PEDF overexpression in tumor cells similarly increased TSP-1 expression, with transcription factors implicated in this coordinated regulation. ${ }^{34,37}$ However, the results from this study suggest that their respective roles in mediating tissue repair are unique. This is suggested by protein structure interactions with the tissue matrix and the phenotype of transgenic mice. Both proteins share binding affinity for glycosaminoglycans, such as heparin. TSP-1's heparin-binding domain stimulates focal adhesion disassembly and confers survival properties to fibroblasts after detachment from the matrix. ${ }^{38}$ Heparin accelerates PEDF's proteolytic degradation. ${ }^{39}$ The absence of TSP-1 in murine models has not been associated with an overt stromal phenotype unless injury studies are undertaken. ${ }^{40}$ In wound injury models, TSP-1-null mice display impaired wound healing and decreased levels of active 
TGF- $\beta 1$ at incision sites. ${ }^{40}$ The loss of PEDF in mice, however, confers marked baseline stromal abnormalities noted in the current and previous studies. ${ }^{6,9}$ These data indicate that both TSP-1 and PEDF play regulatory roles in maintaining the integrity of normal and injured stromal responses that are distinct from their shared actions as inhibitors of angiogenesis.

Little is known about PEDF's role in the normal pancreas. Acinar cell proliferation and stromal expansion were noted in the pancreas of PEDF-null mice but was not further characterized. ${ }^{6}$ More is known about the role of PEDF in pancreatic ductal adenocarcinoma (PDAC). In human PDAC specimens, loss of PEDF staining was associated with metastases and decreased survival compared with PDAC with retained PEDF. ${ }^{41}$ PEDF treatments inhibited PDAC growth and decreased peritoneal spread in mice. ${ }^{42}$ Although these studies highlighted PEDF's known anti-angiogenic effects, the tumor-promoting role of stromal cells in PDAC growth suggests that PEDF's role as a negative regulator of fibroblast activation may also contribute to these antitumor effects. ${ }^{43}$ Thus, PEDF's ability to inhibit multiple cellular compartments makes it a potential therapeutic agent for fibrotic and neoplastic diseases involving the pancreas.

The findings reported herein also highlight differences seen with PEDF expression in the liver after ethanol feeding. ${ }^{9}$ Researchers ${ }^{9,10}$ previously noted abundant liver expression of PEDF in normal rodent and human livers that decreased with 6 weeks of ethanol feeding or tissue fibrosis. Similar to the findings noted in the pancreas, liver PEDF gene expression actually increased with ethanol feeding, but the induction of proteases led to its proteolytic degradation, resulting in depleted PEDF protein levels by immunoblotting and staining. ${ }^{9}$ Unlike the liver, however, the pancreatic parenchyma had relatively scattered and faint labeling for PEDF at baseline. (A notable exception was the uniformly intense labeling of islet cells.) These differences likely reflect tissue-specific PEDF gene expression, whereby the liver had the highest expression levels, whereas those in the pancreas were low. ${ }^{44}$ Other studies ${ }^{7,45,46}$ indicate significant PEDF production by organs, such as the kidney and adipose tissue, and suggest that an increased distribution may occur under inflammatory conditions, such as obesity. Given PEDF's role in ameliorating tissue fibrosis, the contribution of other organs may be reflected in circulating systemic levels. Future studies measuring serum PEDF levels at various stages of fibrosis may help to shed additional information on PEDF's role in mitigating tissue fibrosis.

The findings detailing PEDF's effects on pancreatic fibrosis in this study support other studies that have identified PEDF's ability to modulate the extracellular matrix. Although PEDF's effect on TSP-1 is one notable effect, additional mechanisms underlying PEDF's ability to maintain the integrity of the extracellular matrix remain to be investigated. A recent study ${ }^{47}$ identified PEDF as an endogenous antagonist of LRP6, a coreceptor for the canonical Wnt- $\beta$-catenin signaling pathway. In the liver, constitutive activation of Wnt- $\beta$ - catenin signaling yielded defective hepatocyte differentiation but fully developed bile ducts that led to remodeling of the ductal plate. ${ }^{48}$ Blockade of the Wnt pathway, moreover, can restore the normal liver matrix by inhibiting hepatic stellate cell activation. ${ }^{49}$ Thus, future studies evaluating PEDF's role within the context of its ability to modulate Wnt- $\beta$-catenin signaling may further elucidate PEDF's biological effects.

In summary, PEDF expression was induced in the wildtype pancreas after pancreatitis. The absence of PEDF, however, predisposed to early pancreatic fibrosis but did not impede the resolution of pancreatic scarring at later time points. Consistent with this tissue injury pattern, the absence of PEDF was associated with increased expression of multiple profibrogenic cytokines at baseline. Significantly, the angiogenic inhibitors PEDF and TSP-1 are reciprocally regulated and reflect their respective functions in maintaining homeostasis in the pancreatic stromal compartment.

\section{References}

1. Witt $H$, Apte MV, Keim V, Wilson JS: Chronic pancreatitis: challenges and advances in pathogenesis, genetics, diagnosis, and therapy. Gastroenterology 2007, 132:1557-1573

2. Omary MB, Lugea A, Lowe AW, Pandol SJ: The pancreatic stellate cell: a star on the rise in pancreatic diseases. J Clin Invest 2007, 117:50-59

3. Haber PS, Keogh GW, Apte MV, Moran CS, Stewart NL, Crawford DH, Pirola RC, McCaughan GW, Ramm GA, Wilson JS: Activation of pancreatic stellate cells in human and experimental pancreatic fibrosis. Am J Pathol 1999, 155:1087-1095

4. Pignolo RJ, Cristofalo VJ, Rotenberg MO: Senescent WI-38 cells fail to express EPC-1, a gene induced in young cells upon entry into the G0 state. J Biol Chem 1993, 268:8949-8957

5. Pignolo RJ, Francis MK, Rotenberg MO, Cristofalo VJ: Putative role for EPC-1/PEDF in the G0 growth arrest of human diploid fibroblasts. J Cell Physiol 2003, 195:12-20

6. Doll JA, Stellmach VM, Bouck NP, Bergh AR, Lee C, Abramson LP, Cornwell ML, Pins MR, Borensztajn J, Crawford SE: Pigment epithelium-derived factor regulates the vasculature and mass of the prostate and pancreas. Nat Med 2003, 9:774-780

7. Wang JJ, Zhang SX, Lu K, Chen Y, Mott R, Sato S, Ma JX: Decreased expression of pigment epithelium-derived factor is involved in the pathogenesis of diabetic nephropathy. Diabetes 2005, 54:243-250

8. Wang JJ, Zhang SX, Mott R, Knapp RR, Cao W, Lau K, Ma JX Salutary effect of pigment epithelium-derived factor in diabetic nephropathy: evidence for antifibrogenic activities. Diabetes 2006 , 55:1678-1685

9. Chung C, Shugrue C, Nagar A, Doll JA, Cornwell M, Gattu A, Kolodecik T, Pandol SJ, Gorelick F: Ethanol exposure depletes hepatic pigment epithelium-derived factor, a novel lipid regulator. Gastroenterology 2009, 136:331.e2-340.e2

10. Ho TC, Chen SL, Shih SC, Wu JY, Han WH, Cheng HC, Yang SL, Tsao YP: Pigment epithelium-derived factor is an intrinsic antifibrosis factor targeting hepatic stellate cells. Am J Pathol 2010, 177:1798-1811

11. Andreu-Agullo C, Morante-Redolat JM, Delgado AC, Farinas I: Vascular niche factor PEDF modulates Notch-dependent stemness in the adult subependymal zone. Nat Neurosci 2009, 12:1514-1523

12. Ramirez-Castillejo C, Sanchez-Sanchez F, Andreu-Agullo C, Ferron SR, Aroca-Aguilar JD, Sanchez P, Mira H, Escribano J, Farinas I: Pigment epithelium-derived factor is a niche signal for neural stem cell renewal. Nat Neurosci 2006, 9:331-339

13. Dawson DW, Volpert OV, Gillis P, Crawford SE, Xu H, Benedict W, Bouck NP: Pigment epithelium-derived factor: a potent inhibitor of angiogenesis. Science 1999, 285:245-248

14. Tombran-Tink J, Barnstable CJ: PEDF: a multifaceted neurotrophic factor. Nat Rev Neurosci 2003, 4:628-636

15. Petersen SV, Valnickova Z, Enghild JJ: Pigment-epithelium-derived factor (PEDF) occurs at a physiologically relevant concentration in 
human blood: purification and characterization. Biochem J 2003, 374:199-206

16. Pollina EA, Legesse-Miller A, Haley EM, Goodpaster T, RandolphHabecker J, Coller HA: Regulating the angiogenic balance in tissues: a potential role for the proliferative state of fibroblasts. Cell Cycle 2008, 7:2056-2070

17. Pignolo RJ, Rotenberg MO, Cristofalo VJ: Analysis of EPC-1 growth state-dependent expression, specificity, and conservation of related sequences. J Cell Physiol 1995, 162:110-118

18. Ueda S, Yamagishi S, Matsui T, Jinnouchi Y, Imaizumi T: Administration of pigment epithelium-derived factor inhibits left ventricular remodeling and improves cardiac function in rats with acute myocardial infarction. Am J Pathol 2011, 178:591-598

19. Park K, Jin J, Hu Y, Zhou K, Ma JX: Overexpression of pigment epithelium-derived factor inhibits retinal inflammation and neovascularization. Am J Pathol 2011, 178:688-698

20. Samkharadze T, Erkan M, Reiser-Erkan C, Demir IE, Kong B, Ceyhan GO, Michalski CW, Esposito I, Friess H, Kleeff J: Pigment epitheliumderived factor associates with neuropathy and fibrosis in pancreatic cancer. Am J Gastroenterol 2011, 106:968-980

21. Bornstein P: Thrombospondins as matricellular modulators of cell function. J Clin Invest 2001, 107:929-934

22. Neuschwander-Tetri BA, Talkad V, Otis Stephen F: Induced thrombospondin expression in the mouse pancreas during pancreatic injury. Int J Biochem Cell Biol 2006, 38:102-109

23. Crawford SE, Stellmach V, Murphy-Ullrich JE, Ribeiro SM, Lawler J, Hynes RO, Boivin GP, Bouck N: Thrombospondin-1 is a major activator of TGF-beta1 in vivo. Cell 1998, 93:1159-1170

24. Murphy-Ullrich JE, Poczatek M: Activation of latent TGF-beta by thrombospondin-1: mechanisms and physiology. Cytokine Growth Factor Rev 2000, 11:59-69

25. Belmadani S, Bernal J, Wei CC, Pallero MA, Dell'italia L, MurphyUllrich JE, Berecek KH: A thrombospondin-1 antagonist of transforming growth factor-beta activation blocks cardiomyopathy in rats with diabetes and elevated angiotensin II. Am J Pathol 2007, 171:777-789

26. Daniel C, Wiede J, Krutzsch HC, Ribeiro SM, Roberts DD, MurphyUllrich JE, Hugo C: Thrombospondin-1 is a major activator of TGFbeta in fibrotic renal disease in the rat in vivo. Kidney Int 2004 65:459-468

27. Ribeiro SM, Poczatek M, Schultz-Cherry S, Villain M, Murphy-Ullrich JE: The activation sequence of thrombospondin-1 interacts with the latency-associated peptide to regulate activation of latent transforming growth factor-beta. J Biol Chem 1999, 274:13586-13593

28. Sweetwyne MT, Pallero MA, Lu A, Van Duyn Graham L, MurphyUllrich JE: The calreticulin-binding sequence of thrombospondin 1 regulates collagen expression and organization during tissue remodeling. Am J Pathol 2010, 177:1710-1724

29. Tsukamoto H, French SW: Evolution of intragastric ethanol infusion model. Alcohol 1993, 10:437-441

30. Van Laethem JL, Robberecht P, Resibois A, Deviere J: Transforming growth factor beta promotes development of fibrosis after repeated courses of acute pancreatitis in mice. Gastroenterology 1996, 110: 576-582

31. Ulmasov B, Xu Z, Tetri LH, Inagami T, Neuschwander-Tetri BA: Protective role of angiotensin II type 2 receptor signaling in a mouse model of pancreatic fibrosis. Am J Physiol Gastrointest Liver Physiol 2009, 296:G284-G294

32. Xie Z, Singh M, Singh K: Differential regulation of matrix metalloproteinase-2 and -9 expression and activity in adult rat cardiac fibroblasts in response to interleukin-1beta. J Biol Chem 2004, 279: 39513-39519

33. Xue M, March L, Sambrook PN, Jackson CJ: Differential regulation of matrix metalloproteinase 2 and matrix metalloproteinase 9 by acti- vated protein C: relevance to inflammation in rheumatoid arthritis. Arthritis Rheum 2007, 56:2864-2874

34. Guan M, Pang CP, Yam HF, Cheung KF, Liu WW, Lu Y: Inhibition of glioma invasion by overexpression of pigment epithelium-derived factor. Cancer Gene Ther 2004, 11:325-332

35. Viloria-Petit A, Miquerol L, Yu JL, Gertsenstein M, Sheehan C, May L, Henkin J, Lobe C, Nagy A, Kerbel RS, Rak J: Contrasting effects of VEGF gene disruption in embryonic stem cell-derived versus oncogene-induced tumors. EMBO J 2003, 22:4091-4102

36. Friedman SL: Cytokines and fibrogenesis. Semin Liver Dis 1999, 19:129-140

37. Uchida $H$, Hayashi $H$, Kuroki M, Uno K, Yamada $H$, Yamashita $Y$, Tombran-Tink J, Oshima K: Vitamin A up-regulates the expression of thrombospondin-1 and pigment epithelium-derived factor in retinal pigment epithelial cells. Exp Eye Res 2005, 80:23-30

38. Murphy-Ullrich JE: The de-adhesive activity of matricellular proteins: is intermediate cell adhesion an adaptive state? J Clin Invest 2001, 107:785-790

39. Valnickova Z, Petersen SV, Nielsen SB, Otzen DE, Enghild JJ: Heparin binding induces a conformational change in pigment epitheliumderived factor. J Biol Chem 2007, 282:6661-6667

40. Agah A, Kyriakides TR, Lawler J, Bornstein P: The lack of thrombospondin-1 (TSP1) dictates the course of wound healing in doubleTSP1/TSP2-null mice. Am J Pathol 2002, 161:831-839

41. Uehara $\mathrm{H}$, Miyamoto $\mathrm{M}$, Kato K, Ebihara $\mathrm{Y}$, Kaneko $\mathrm{H}$, Hashimoto $\mathrm{H}$, Murakami Y, Hase R, Takahashi R, Mega S, Shichinohe T, Kawarada $\mathrm{Y}$, Itoh T, Okushiba S, Kondo S, Katoh H: Expression of pigment epithelium-derived factor decreases liver metastasis and correlates with favorable prognosis for patients with ductal pancreatic adenocarcinoma. Cancer Res 2004, 64:3533-3537

42. Hase R, Miyamoto $M$, Uehara $H$, Kadoya M, Ebihara $Y$, Murakami $Y$, Takahashi R, Mega S, Li L, Shichinohe T, Kawarada Y, Kondo S: Pigment epithelium-derived factor gene therapy inhibits human pancreatic cancer in mice. Clin Cancer Res 2005, 11:8737-8744

43. Vonlaufen A, Joshi S, Qu C, Phillips PA, Xu Z, Parker NR, Toi CS, Pirola RC, Wilson JS, Goldstein D, Apte MV: Pancreatic stellate cells: partners in crime with pancreatic cancer cells. Cancer Res 2008, 68:2085-2093

44. Tombran-Tink J, Mazuruk K, Rodriguez IR, Chung D, Linker T, Englander E, Chader GJ: Organization, evolutionary conservation, expression and unusual Alu density of the human gene for pigment epithelium-derived factor, a unique neurotrophic serpin. Mol Vis 1996, 2:11

45. Crowe S, Wu LE, Economou C, Turpin SM, Matzaris M, Hoehn KL, Hevener AL, James DE, Duh EJ, Watt MJ: Pigment epithelium-derived factor contributes to insulin resistance in obesity. Cell Metab 2009, 10:40-47

46. Yamagishi S, Adachi H, Abe A, Yashiro T, Enomoto M, Furuki K, Hino A, Jinnouchi $Y$, Takenaka K, Matsui T, Nakamura K, Imaizumi T: Elevated serum levels of pigment epithelium-derived factor in the metabolic syndrome. J Clin Endocrinol Metab 2006, 91:2447-2450

47. Park K, Lee K, Zhang B, Zhou T, He X, Gao G, Murray AR, Ma JX: Identification of a novel inhibitor of the canonical Wnt pathway. Mol Cell Biol 2011, 31:3038-3051

48. Decaens T, Godard C, de Reynies A, Rickman DS, Tronche F, Couty JP, Perret C, Colnot S: Stabilization of beta-catenin affects mouse embryonic liver growth and hepatoblast fate. Hepatology 2008, 47: 247-258

49. Cheng JH, She H, Han YP, Wang J, Xiong S, Asahina K, Tsukamoto $\mathrm{H}$ : Wnt antagonism inhibits hepatic stellate cell activation and liver fibrosis. Am J Physiol Gastrointest Liver Physiol 2008, 294:G39-G49 